\title{
Cyclin-dependent kinase 9 may as a novel target in downregulating the atherosclerosis inflammation (Review)
}

\author{
YEMING HAN $^{1}$, YANG ZHAN ${ }^{2}$, GUIHUA HOU ${ }^{2}$ and LI LI ${ }^{1}$ \\ ${ }^{1}$ Key Laboratory of Cardiovascular Remodeling and Function Research, Chinese Ministry of Education \\ and Chinese Ministry of Public Health, Qilu Hospital, Shandong University; ${ }^{2}$ Institute of Experimental \\ Nuclear Medicine, School of Medicine, Shandong University, Jinan, Shandong 250012, P.R. China
}

Received June 9, 2014; Accepted July 17, 2014

DOI: $10.3892 /$ br.2014.322

\begin{abstract}
Inflammation is a key component of atherosclerosis. Genes coding for inflammatory or anti-inflammatory molecules are considered good candidates for estimating the risk of developing atherosclerosis. Cyclin-dependent kinase 9 (CDK9), the kinase of positive transcription elongation factor $b$ (P-TEFb), is crucial in the cell cycle and apoptosis. Previous studies have focused on its inhibition of immune cells for the resolution of inflammation. Considering the effects of inflammation in the pathogenicity of atherosclerosis, decreasing inflammation through the inhibition of CDK9 may be useful for the prognosis of atherosclerosis. The aim of this review was to examine whether inhibition of the CDK9 monocyte may affect the process of inflammation by acting on the cytokine secretion and interacting with endothelial cells (ECs). Thus, CDK9 may be a novel target for the diagnosis and therapy of atherosclerosis.
\end{abstract}

\section{Contents}

1. Introduction

2. CDK9 and P-TEFb

3. CDK9 with $\mathrm{T}$ cells and monocytes

4. CDK9 and endothelium

5. CDK9 and inflammatory cytokines

Correspondence to: Professor $\mathrm{Li} \mathrm{Li}$, Key Laboratory of Cardiovascular Remodeling and Function Research, Chinese Ministry of Education and Chinese Ministry of Public Health, Qilu Hospital, Shandong University, 107 Wenhua West Road, Jinan, Shandong 250012, P.R. China

E-mail: lilihospital@163.com

Key words: cyclin-dependent kinase 9, atherosclerosis, positive transcription elongation factor $\mathrm{b}$, inflammation, immunoregulation

\section{Introduction}

Atherosclerosis is a complex vascular disease that usually begins in the first decade of life and is now recognized mainly as an inflammatory illness (1). It is a process characterized by the accumulation of lipids, mononuclear cells, fibrous components and calcium in the arteries (2). Vascular injury, recruitment of monocyte cells and infiltration of foam cells in combination with $\mathrm{T}$ lymphocytes, promotes lesion formation $(2,3)$.

Atherogenesis was traditionally considered a metabolic disease demonstrating arterial obstruction by fatty deposits in its wall (4). The current view is that atherogenesis involves highly specific biochemical and molecular responses with continuous interactions between different cellular players. Despite the presence of inflammatory reaction in each individual step of atherosclerosis from its beginning to a terminal manifestation, the cause-effect relationship of the two processes remains to be elucidated (4).

Inflammation is crucial in the pathogenesis of atherosclerosis. Genes coding for inflammatory or anti-inflammatory molecules are considered potentially ideal candidates for estimating the risk of developing atherosclerosis (5-7). Accordingly, the production of high inflammatory molecules has been associated with atherosclerosis (8-11). Positive control of inflammation may play a protective role against atherosclerosis and is a potential therapeutic candidate for the prevention of atheroma formation.

Cyclin-dependent kinases (CDKs) play an important role in the cell cycle and apoptosis. An increasing number of agents have been identified to interfere with the pathogenicity of atherosclerosis by targeting CDKS. Of these, CDK9 has been shown to exhibit marked characteristics in controlling inflammation (12). In a previous study (unpublished data), results of the 2-D proteomics analysis revealed that CDK9 was highly expressed in the serum of patients with atherosclerosis. The aim of the study was to analyze those results and identify the key role for CDK9 and its underlying mechanism.

\section{CDK9 and P-TEFb}

The CDK family can be divided into two major functional groups based on their roles in the cell cycle and/or transcriptional control. CDK9 associates with each of four cycles 
(T1, T2a, T2b and K), forming distinct positive transcription elongation factors (P-TEFs). Findings of previous studies (13) have demonstrated that $\mathrm{P}-\mathrm{TEFb}$ is positively regulated with transcriptional elongation by phosphorylating the C-terminal domain (CTD) of RNA polymerase II (RNA pol II), as well as negative elongation factors, which block elongation by RNA pol II shortly after the initiation of transcription. P-TEFb has also been found to positively modulate other steps during transcription. In addition, 'abnormal' CDK9 function is associated with certain diseases. Specifically, activity of the cyclin T1/CDK9 complex is essential for human immunodeficiency virus (HIV)-1 replication and CDK9 upregulation is associated with cardiac hypertrophy (13-17).

As opposed to other CDKs, CDK9 does not control the cell cycle. Instead it promotes RNA synthesis in genetic programmes for cell growth, differentiation and viral pathogenesis (18). CDK9 inhibition appears to contribute to the anticancer activity of the majority of CDK inhibitors under clinical investigation. CDK9 was identified in investigations conducted on HIV as retroviruses hijack host transcription (19) and CDK9 inhibitors may serve as specific antiretroviral agents, particularly due to their involvement in preventing drug resistance. Myocardial hypertrophy is a risk factor for congestive heart failure that is characterized by the derepression of CDK9 activity (20). Therefore, CDK9 inhibitors may serve a therapeutic role in cardiology. Despite indications that CDK9 inhibition may be utilized as a therapeutic strategy in all three indications, the lack of selective inhibitors has confounded clinical development (21).

For P-TEFb, which is involved in the transcriptional elongation phase, the processing of transcription is arrested due to blockage by negative transcription elongation factors (22). Owing to additional phosphorylation in the RNA pol II CTD at serine 2 by CDK 9 activity, P-TEFb enables pol II to resume transcription subsequent to the promoter-proximal pause. In addition to RNA pol II, P-TEFb phosphorylates transcriptional complexes including the 5,6-dichloro-1- $\beta$-D-ribofuranosylbenzimidazole sensitivity-inducing factor and the negative elongation factor which are responsible for the promoter-proximal pausing of transcriptional machinery (20). P-TEFb primarily functions as in the elongation step, however, it has been identified in the pre-initiation transcription complex. Although CDK9 and cyclin T1 individually do not possess any DNA-binding activity, the recruitment of $\mathrm{P}-\mathrm{TEFb}$ to promoters is critical to activate gene expression (20). DNA sequence-specific transcription factors, such as nuclear factor $\kappa \mathrm{B}(\mathrm{NF}-\kappa \mathrm{B}), \mathrm{c}-\mathrm{myc}, \mathrm{c}$-fos and peroxisome proliferator-activated receptor $\gamma$, have been designated as $\mathrm{P}-\mathrm{TEFb}$-associated factors that recruit $\mathrm{P}-\mathrm{TEFb}$ to their respective gene promoters.

\section{CDK9 with T cells and monocytes}

Stimulation of primary human $\mathrm{T}$ lymphocytes results in the upregulation of cyclin T1 expression, which correlates with phosphorylation of the CTD of RNA pol II. The upregulation of cyclin T1 and concomitant stabilization of CDK9 may facilitate the productive replication of HIV in activated T cells (23). It has been reported that the treatment of peripheral blood lymphocytes with the mitogens, phytohaemagglutinin
(PHA) and phorbol myristate acetate (PMA), results in the accumulation of cyclin T1 through distinct mechanisms. PHA leads to the accumulation of cyclin T1 mRNA and protein, which results from cyclin T1 mRNA stabilization, with no significant alteration in cyclin $\mathrm{T} 1$ promoter activity (23). Cyclin T1 mRNA stabilization necessitates the activation of calcineurin and c-Jun N-terminal kinase as inhibition of either prevents cyclin T1 accumulation. By contrast, PMA leads to cyclin $\mathrm{T} 1$ protein upregulation through the stabilization of cyclin $\mathrm{T} 1$ protein, apparently independently of the proteasome and without accumulation of cyclin T1 mRNA. This process is a function of $\mathrm{Ca}^{2+}$-independent protein kinase $\mathrm{C}$ activity. However, extracellular-signal-regulated kinases $1 / 2$ activation is not necessary. The PHA and anti-CD3 antibodies induced the expression of the cyclin/CDK complexes involved in RNA pol II CTD phosphorylation and the G1-S cyclins regulating cell cycle progression (23). By contrast, PMA alone does not induce the expression of G1-S cyclins although it is often as potent as PHA in inducing RNA pol II cyclin/CDK complexes. These findings suggest the expression and activation of RNA pol II kinases is coordinated by pathways that independently stimulate gene expression although they are insufficient to induce S-phase entry in primary T cells (23).

In human cells, CDK9 in the cycT1/P-TEFb complex is required for transcription of major histocompatibility class II (MHC II) genes. However, which human genes require $\mathrm{P}-\mathrm{TEFb}$ function in vivo remains to be determined. Overexpression of a dominant negative CDK9 protein in activated Jurkat T cells does not affect the induction of CD25, CD69, or interleukin-2 (IL-2), three molecules known to be important for T-cell function (24). By contrast, the overexpression of a dominant negative CDK9 protein in the U937 promonocytic cell line induced cell sensitivity to apoptosis, particularly after PMA treatment in order to induce differentiation, suggesting that CDK9 has an antiapoptotic function during monocyte differentiation (24). The elucidation of normal cell functions of CDK9 in CD4+ $\mathrm{T}$ cells and monocytes/macrophages is therefore vital for assessing the feasibility of CDK9 as a therapeutic target (25).

The ability to readily undergo apoptosis is important for monocyte homeostasis, since monocytes usually circulate in the blood for a period of only a few days, during which time they emigrate to tissues and differentiate to macrophages or die through apoptosis $(26,27)$.

Findings of previous studies have shown that CDK9 catalytic activity is low in promonocytic cells due to limiting amounts of the cycT1 regulatory subunit $(28,29)$, suggesting that a low level of cycT1 protein in monocytes and thus a low level of CDK9 function, may be required for apoptosis in the absence of differentiation. The antiapoptotic function of CDK9 may be due to involvement in an apoptotic pathway or blocking in the differentiation program of monocytes by the dominant negative CDK9 protein.

Results of a previous study on monocyte differentiation suggested that cells have an intrinsic program to differentiate when apoptosis becomes an obstacle in enforcing the expression of B-cell lymphoma 2 (30). This finding suggests that CDK9 functions in the $\mathrm{P}-\mathrm{TEFb}$ complex to organize gene transcription, such as that for $p 21 W A F 1$, whose expression confers resistance to apoptosis prior to monocyte differentiation (31). An additional possibility is that CDK9 function is 
necessary for the differentiation program of monocytes. The results suggest that $\mathrm{CDK} 9$ function is likely to be crucial to the monocyte/macrophage life cycle. As CDK9 is important in monocyte apoptosis and differentiation, it may be a feasible therapeutic target in the treatment of atherosclerosis (32). The effect of CDK9 on the transcription and extravasation of leukocyte to promote resolution of inflammation has been previously investigated $(24,33)$.

CDK9-deficient mice would be the optimal model to determine the exact role of CDK9 in inflammatory processes in vivo. However, such a model is not available. Thus, current investigations depend on the use of kinase inhibitors (34). The overactivity of cell cycle CDKs is evident in tumor cells, leading to their growth advantage. Consequently, CDK inhibition has been suggested as a novel anticancer strategy (35). Flavopiridol and Roscovitine are the most intensively studied CDK inhibitors. Flavopiridol was found to have potent antiproliferative action on 60 human cancer cell lines in the US National Cancer Institute screen panel and was the first CDK inhibitor to be submitted to clinical trials (36). Flavopiridol suppressed inflammatory processes such as inhibtion of murine collagen-induced arthritis in a dose-dependent manner (37). Additionally, it effectively limited murine hepatitis induced by concanavalin A (Con A), which triggers a strong and rapid leukocyte-dependent inflammatory liver injury (38). Roscovitine protected against acute graft-versus-host disease by blocking the clonal expansion of T cells (12) and against glomerulonephritis by inhibiting the proliferative response of $\mathrm{T}$ and $\mathrm{B}$ cells (39). Thus, the anti-inflammatory effect of CDKs inhibitors has been ascribed to its inhibition of the proliferation and induction of cell death in immune cells and. Consequently, recent investigations have focused on these actions in the context of resolution of inflammation (40).

\section{CDK9 and endothelium}

CDK inhibitors were suggested to induce immune cell death and thereby increase the resolution of inflammation. Notably, beyond the antiproliferative actions and cell death induction of CDK9, to the best of our knowledge, few studies have focused on the action of CDK inhibitors, at an early and crucial step in inflammation, and the interaction of leukocytes with endothelial cells (ECs). Excessive leukocyte extravasation from the blood into the tissue, a process that is closely regulated by the endothelium, is a hallmark of inflammation and contributes to the pathogenesis and progression of numerous severe inflammation-associated pathologies, including atherosclerosis, arthritis, or asthma. In mice, hepatitis was identified by intravenous application of ConA (41). Liver injury was greatly suppressed by pretreatment with flavopiridol. Additionally, a significant decrease of the endothelium-specific adhesion molecule E-selectin and the intercellular adhesion molecule-1 mRNA expression in flavopiridol-treated mice was observed. The abovementioned findings show that the anti-inflammatory action of flavopiridol is associated with the reduction of leukocyte infiltration and cell adhesion molecule expression which emphasize the inhibition of CDK9 as an interesting approach against inflammation-associated pathologies. CDK9 inhibition is considered to have potential value in the combat against inflammation (12). Independent of its function as a subunit of
$\mathrm{P}-\mathrm{TEFb}, \mathrm{CDK} 9$ is considered to interact with $\kappa \mathrm{B}$ p65 and to repress $\mathrm{NF}-\kappa$ cytoplasmic regions of gp130, the receptor for the proinflammatory cytokine IL-6. I has also been reported that CDK9 inhibitors disrupt IL-6/STAT3 signaling in the liver (42). HEXIM1, an endogenous inhibitor of P-TEFb, is associated with $\mathrm{NF}-\kappa \mathrm{B}$ dependent gene transcription in smooth muscle cells. Authors of that study (12) assessed the action of CDK9 suppression on leukocyte-EC interaction and, in particular, on endothelial activation, an important inflammation-triggering event. Low-dose flavopiridol effectively protects against inflammation-induced interactions between leukocytes and the endothelium, primarily by blocking endothelial activation. This occurs as $\kappa \mathrm{B}$ does not change the inhibition B activation cascade. Notably, the canonical NF- $\kappa \mathrm{B}$ of the proinflammatory transcription factor $\mathrm{NF}-\kappa \mathrm{B}$ flavopiridol $\mathrm{B}$ induced $\kappa$, while $\mathrm{NF}-\kappa \mathrm{B}$ transcription was decreased via CDK9 suppression. Thus, the data suggest flavopiridol as a promising anti-inflammatory agent. Moreover, CDK9 inhibition is revealed as an interesting approach to the treatment of inflammation-associated diseases (43-47).

\section{CDK9 and inflammatory cytokines}

Cytokines play a key role during the process of inflammation. IL-6 and IL-8 are regarded as inducers of alteration in endothelial functions. A number of studies have reported that infection by the dengue virus (DENV) induces the secretion of IL-8. In addition to cellular proteins, P-TEFb interacts with viral gene products to assist the progress of the viral life cycle. It has been reported that $\mathrm{P}-\mathrm{TEFb}$ is crucial for DENV-induced IL-8 mRNA expression (48). The association between P-TEFb and DENV core protein leads to an increase in the activity of $N F-\kappa B$ and the upregulation of $I L-8$ gene transcription. Recruitment of P-TEFb and DENV core proteins to the IL- 8 promoter suggested that the association of P-TEFb with DENV core protein is involved in IL-8 induction by DENV infection. $\mathrm{P}-\mathrm{TEFb}$ has been identified as a key co-activator that associates with DENV core protein to induce IL-8 expression when DENV infects host cells (48). P-TEFb is importan for DENV induced IL-8 expression and this IL-8 production is dependent on DENV core protein and the activation of $\mathrm{NF}-\kappa \mathrm{B}$ element within the IL-8 gene promoter. The abovementioned findings showed the recruitment of P-TEFb and DENV core protein to the IL- 8 promoter, suggesting that the association of $\mathrm{P}-\mathrm{TEFb}$ with DENV core protein is crucial for triggering the induction of IL- 8 expression. The core of the IL- 8 promoter is located at -1 to -133 within the 5 flanking region of the $I L-8$ gene. It is indispensable and sufficient for transcriptional regulation of the gene. Several transcriptional activators, including NF- $\kappa \mathrm{B}$ and activator protein 1 are present in this core region (48).

IL-10 is produced by a number of cells such as $\mathrm{T}$ helper (Th)1, Th2, Th17, T regulatory, CD8+ T cells, B cells and myeloid cells. One of the primary functions of IL-10 is the regulation of pathogen-mediated activation of macrophages and dendritic cells. IL-10 suppresses the T cell-activating potential of APC by downregulating MHC class II and the expression of costimulatory molecules such as CD80 and CD86. IL-10 also inhibits the expression of chemokines, inflammatory enzymes and potent proinflammatory cytokines such as tumor necrosis factor $\alpha(\mathrm{TNF} \alpha)$, the target for various clinical strate- 
gies in rheumatoid arthritis and Crohn's disease (49). Although recent advances have resulted in a greater understanding of the regulation of IL-10 production, the precise mechanism of IL-10-dependent inhibition of TNF $\alpha$ remains to be elucidated. Activation of STAT3, as a consequence of IL-10 binding to its cell surface receptor (IL-10R1/IL-10R2), is critical for mediating the anti-inflammatory response. IL-10 has been shown to target the transcriptional and post-transcriptional processes in a gene-specific manner (49).

IL-10 plays a non-redundant role in limiting inflammation in vivo. Using primary human macrophages, IL-10 has been shown to suppress selected inflammatory genes, primarily at the transcription level. For the TNF gene, this occurs, not through the inhibition of RNA pol II recruitment and transcription initiation, but through a mechanism for targeting the stimulation of transcription elongation by CDK9. The region downstream of the TNF untranslated region containing the NF- $\kappa B$ binding motif, both for the induction of transcription by lipopolysaccharide and its inhibition by IL-10, is crucial (50). Deregulation of the inflammatory response can lead to diseases such as rheumatoid arthritis, Crohn's disease, or septic shock. The cytokine IL-10 limits the magnitude, duration and detrimental outcome of the inflammatory response. This finding was identified in the generation of IL-10-deficient mice that spontaneously develop inflammatory bowel disease and with the identification of patients with homozygous mutations in the IL-10 receptor subunits who present with early-onset colitis (50).

Based on above findings, we hypothesized that the transcriptional inhibition of monocytes, with CDK9 inhibition, may influence the secretion of inflammatory cytokines, monocyte differentiation and interaction of monocytes with endothelium, inhibiting monocyte infiltration by direct involvement on endothelial activation, which may decrease the pathologic effect of atherosclerosis. Future studies are to focus on monocyte and its interference with CDK9 and detection of its effect on monocyte proliferation and apoptosis. The underlying molecular mechanisms with a special focus on the signaling processes in inflammation-activated monocytes are also to be investigated. Findings of the present review may contribute to understanding the role of CDK9 in the inflammatory response, as well as be useful in identifying new targets of anti-atherosclerosis in the clinic.

\section{References}

1. García-Gómez C, Bianchi M, de la Fuente D, Badimon L, Padró T, Corbella E and Pintó X: Inflammation, lipid metabolism and cardiovascular risk in rheumatoid arthritis: A qualitative relationship? World J Orthop 5: 304-311, 2014.

2. Randolph GJ: Mechanisms that regulate macrophage burden in atherosclerosis. Circ Res 114: 1757-1771, 2014.

3. Ait-Oufella H, Sage AP, Mallat Z and Tedgui A: Adaptive (T and B cells) immunity and control by dendritic cells in atherosclerosis. Circ Res 114: 1640-1660, 2014.

4. Mangoni AA: Folic acid, inflammation, and atherosclerosis: false hopes or the need for better trials? Clin Chim Acta 367: 11-19, 2006

5. Incalcaterra E, Accardi G, Balistreri CR, Caimi G, Candore G Caruso $\mathrm{M}$ and Caruso C: Pro-inflammatory genetic markers of atherosclerosis. Curr Atheroscler Rep 15: 329-335, 2013.

6. Raman K, Chong M, Akhtar-Danesh GG, D'Mello M, Hasso R, Ross S, Xu F and Paré G: Genetic markers of inflammation and their role in cardiovascular disease. Can J Cardiol 29: 67-74, 2013.

7. Van-Assche T, Huygelen V, Crabtree MJ and Antoniades C: Gene therapy targeting inflammation in atherosclerosis. Curr Pharm Des 17: 4210-4223, 2011.
8. Lu XT, Zhao YX, Zhang Y and Jiang F: Psychological stress, vascular inflammation, and atherogenesis: potential roles of circulating cytokines. J Cardiovasc Pharmacol 62: 6-12, 2013.

9. Martinet W, Schrijvers DM and De Meyer GR: Molecular and cellular mechanisms of macrophage survival in atherosclerosis. Basic Res Cardiol 107: 297-309, 2012.

10. Di Gregoli K and Johnson JL: Role of colony-stimulating factors in atherosclerosis. Curr Opin Lipidol 23: 412-421, 2012.

11. Chen LJ, Lim SH, Yeh YT, Lien SC and Chiu JJ: Roles of microRNAs in atherosclerosis and restenosis. J Biomed Sci 19: 79-92, 2012.

12. Schmerwitz UK, Sass G, Khandoga AG, et al: Flavopiridol protects against inflammation by attenuating leukocyte-endothelial interaction via inhibition of cyclin-dependent kinase 9. Arterioscler Thromb Vasc Biol 31: 280-288, 2011.

13. Peng J, Zhu Y, Milton JT and Price DH: Identification of multiple cyclin subunits of human P-TEFb. Genes Dev 12: 755-762, 1998.

14. Licastro F, Candore G, Lio D, Porcellini E, Colonna-Romano G, Franceschi $\mathrm{C}$ and Caruso $\mathrm{C}$ : Innate immunity and inflammation in ageing: a key for understanding age-related diseases. Immun Ageing 2: 8, 2005.

15. Stephens JW and Humphries SE: The molecular genetics of cardiovascular disease: clinical implications. J Intern Med 253: 120-127, 2003

16. Lipszyc PS, Cremaschi GA, Zorrilla-Zubilete M, Bertolino ML, Capani F, Genaro AM and Wald MR: Niacin modulates pro-inflammatory cytokine secretion. A potential mechanism involved in its anti-atherosclerotic effect. Open Cardiovasc Med J 7: 90-98, 2013.

17. Baumli S, Hole AJ, Wang LZ, Noble ME and Endicott JA: The CDK9 tail determines the reaction pathway of positive transcription elongation factor b. Cell 20: 1788-1795, 2012.

18. Budhiraja S, Famiglietti M, Bosque A, Planelles V and Rice AP: Cyclin T1 and CDK9 T-loop phosphorylation are downregulated during establishment of HIV-1 latency in primary resting memory CD4 ${ }^{+} \mathrm{T}$ cells. J Virol 87: 1211-1220, 2013.

19. Wang S and Fischer PM: Cyclin-dependent kinase 9: a key transcriptional regulator and potential drug target in oncology, virology and cardiology. Trends Pharmacol Sci 29: 302-313, 2008.

20. Sunagawa Y, Morimoto T, Takaya T, et al: Cyclin-dependent kinase-9 is a component of the p300/GATA4 complex required for phenylephrine-induced hypertrophy in cardiomyocytes. J Biol Chem 285: 9556-9568, 2010.

21. Garriga J and Grana X: Cellular control of gene expression by T-type cyclin/CDK9 complexes. Gene 337: 15-23, 2004.

22. Wang Y, Liu XY and De Clercq E: Role of the HIV-1 positive elongation factor P-TEFb and inhibitors thereof. Mini Rev Med Chem 9: 379-385, 2009.

23. Marshall RM and Grana X: Mechanisms controlling CDK9 activity. Front Biosci 11: 2598-2613, 2006.

24. Foskett SM, Ghose R, Tang DN, Lewis DE and Rice AP: Antiapoptotic function of Cdk9 (TAK/P-TEFb) in U937 promonocytic cells. J Virol 75: 1220-1228, 2001.

25. Fu TJ, Peng J, Lee G, Price DH and Flores O: Cyclin K functions as a CDK9 regulatory subunit and participates in RNA polymerase II transcription. J Biol Chem 274: 34527-34530, 1999.

26. Marshall RM, Salerno D, Garriga J and Grana X: Cyclin T1 expression is regulated by multiple signaling pathways and mechanisms during activation of human peripheral blood lymphocytes. J Immunol 175: 6402-6411, 2005.

27. Kanazawa S, Okamoto T and Peterlin BM: Tat competes with CIITA for the binding to P-TEFb and blocks the expression of MHC class II genes in HIV infection. Immunity 12: 61-70, 2000.

28. Heidenreich S: Monocyte CD14: a multifunctional receptor engaged in apoptosis from both sides. J Leukoc Biol 65: 737-743, 1999.

29. Herrmann CH, Carroll RG, Wei P, Jones KA and Rice AP: Tat-associated kinase, TAK, activity is regulated by distinct mechanisms in peripheral blood lymphocytes and promonocytic cell lines. J Virol 72: 9881-9888, 1998.

30. Miranda MB, Dyer KF, Grandis JR and Johnson DE: Differential activation of apoptosis regulatory pathways during monocytic vs granulocytic differentiation: a requirement for Bcl-X(L)and XIAP in the prolonged survival of monocytic cells. Leukemia 17: 390-400, 2003.

31. Xaus J, Cardo M, Valledor AF, Soler C, Lloberas J and Celada A: Interferon gamma induces the expression of p21waf-1 and arrests macrophage cell cycle, preventing induction of apoptosis. Immunity 11: 103-113, 1999. 
32. Yang X, Gold MO, Tang DN, Lewis DE, Aguilar-Cordova E, Rice AP and Herrmann CH: TAK, an HIV Tat-associated kinase, is a member of the cyclin-dependent family of protein kinases and is induced by activation of peripheral blood lymphocytes and differentiation of promonocytic cell lines. Proc Natl Acad Sci USA 94: 12331-12336, 1997.

33. Leitch AE, Lucas CD, Marwick JA, Duffin R, Haslett C and Rossi AG: Cyclin-dependent kinases 7 and 9 specifically regulate neutrophil transcription and their inhibition drives apoptosis to promote resolution of inflammation. Cell Death Differ 19: 1950-1961, 2012.

34. Berberich N, Uhl B, Joore J, Schmerwitz UK, Mayer BA, Reichel CA, Krombach F, Zahler S, Vollmar AM and Furst R: Roscovitine blocks leukocyte extravasation by inhibition of cyclin-dependent kinases 5 and 9. Br J Pharmacol 163: 1086-1098, 2011.

35. Johnson N and Shapiro GI: Cyclin-dependent kinases (cdks) and the DNA damage response: rationale for cdk inhibitor-chemotherapy combinations as an anticancer strategy for solid tumors. Expert Opin Ther Targets 14: 1199-1212, 2010.

36. Wang LM and Ren DM: Flavopiridol, the first cyclin-dependent kinase inhibitor: recent advances in combination chemotherapy. Mini Rev Med Chem 10: 1058-1070, 2010.

37. Krystof V, Baumli S and Furst R: Perspective of cyclin-dependent kinase 9 (CDK9) as a drug target. Curr Pharm Des 18: 2883-2890, 2012.

38. Sekine C, Sugihara T, Miyake S, Hirai H, Yoshida M, Miyasaka N and Kohsaka H: Successful treatment of animal models of rheumatoid arthritis with small-molecule cyclin-dependent kinase inhibitors. J Immunol 180: 1954-1961, 2008.

39. Li L, Wang H, Kim Js, Pihan G and Boussiotis V: The cyclin dependent kinase inhibitor (R)-roscovitine prevents alloreactive $\mathrm{T}$ cell clonal expansion and protects against acute GvHD. Cell Cycle 8: 1794-1802, 2009.

40. Zoja C, Casiraghi F, Conti S, Corna D, Rottoli D, Cavinato RA, Remuzzi G and Benigni A: Cyclin-dependent kinase inhibition limits glomerulonephritis and extends lifespan of mice with systemic lupus. Arthritis Rheum 56: 1629-1637, 2007.
41. Jia HY, Chen F, Chen JZ, Wu SS, Wang J, Cao QY, Chen Z and Zhu HH: MicroRNA expression profiles related to early stage murine concanavalin A-induced hepatitis. Cell Physiol Biochem 33: 1933-1944, 2014.

42. Hou T, Ray S and Brasier AR: The functional role of an interleukin 6-inducible CDK9.STAT3 complex in human gamma-fibrinogen gene expression. J Biol Chem 282: 37091-37102, 2007.

43. Leitch AE, Haslett C and Rossi AG: Cyclin-dependent kinase inhibitor drugs as potential novel anti-inflammatory and pro-resolution agents. Br J Pharmacol 158: 1004-1016, 2009.

44. Galli SJ, Borregaard N and Wynn TA: Phenotypic and functional plasticity of cells of innate immunity: macrophages, mast cells and neutrophils. Nat Immunol 12: 1035-1044, 2011.

45. Puga I, Cols M, Barra CM, et al: B cell-helper neutrophils stimulate the diversification and production of immunoglobulin in the marginal zone of the spleen. Nat Immunol 13: 170-180, 2011.

46. Gabrilovich DI and Nagaraj S: Myeloid-derived suppressor cells as regulators of the immune system. Nat Rev Immunol 9: $162-174,2009$.

47. Gordon V, Bhadel S, Wunderlich W, et al: CDK9 regulates AR promoter selectivity and cell growth through serine 81 phosphorylation. Mol Endocrinol 24: 2267-2280, 2010.

48. Li LL, Hu ST, Wang SH, Lee HH, Wang YT and Ping YH: Positive transcription elongation factor $\mathrm{b}(\mathrm{P}-\mathrm{TEFb})$ contributes to dengue virus-stimulated induction of interleukin-8 (IL-8). Cell Microbiol 12: 1589-1603, 2010.

49. Smallie T, Ricchetti G, Horwood NJ, Feldmann M, Clark AR and Williams LM: IL-10 inhibits transcription elongation of the human TNF gene in primary macrophages. J Exp Med 207: 2081-2088, 2010.

50. Holgersen K, Kvist PH, Hansen AK and Holm TL: Predictive validity and immune cell involvement in the pathogenesis of piroxicam-accelerated colitis in interleukin-10 knockout mice. Int Immunopharmacol 21: 137-147, 2014. 